\title{
Synthesis and stability of 1,1-dialkyl-1H-azulenium cations
}

Mitsunori Oda, ${ }^{\mathrm{a}, *}$ Nobue Nakajima, ${ }^{\mathrm{b}}$ Nguyen Chung Thanh, ${ }^{\mathrm{b}}$ Takanori Kajioka, ${ }^{\mathrm{b}}$ and Shigeyasu Kuroda ${ }^{\mathrm{b}}$

${ }^{a}$ Department of Chemistry, Faculty of Science, Shinshu University, Asahi 3-1-1, Matsumoto, Nagano 390-8621, Japan

${ }^{\mathrm{b}}$ Department of Applied Chemistry, Faculty of Engineering, University of Toyama, Gofuku 3190, Toyama 930-8555, Japan

Keywords: carbocations, $\mathrm{p} K_{\mathrm{R}}+$ values, tropylium ions, Nazarov cyclizations, intermolecular charge-transfer interaction

*Corresponding author: Tel \& Fax: + 8126337 3343; e-mail: mituoda@shinshu-u.ac.jp

\begin{abstract}
Starting from trimethylsilyl enol ether of 1-acetyl-1,3,5-cycloheptatriene, the title 1,1-dimethyl-, 1,1-diethyl- and 1,1-dipropyl- $1 H$-azulenium cations 6-8 were synthesized in five steps. The order of $\mathrm{p} K_{\mathrm{R}}+$ values of these cations was found to be $\mathbf{7}>\mathbf{8}>\mathbf{6}$. A comparison of the values between 1,1-dialkyl- and 1,1-spiroalkylated $1 H$-azulenium cations with the same number of carbon atoms at the 1-position provided the results of $\mathbf{7}>\mathbf{1}$ and $\mathbf{8}<\mathbf{3}$. The cation $\mathbf{8}$ shows a relatively lower $\mathrm{p} K_{\mathrm{R}}+$ value to those of $\mathbf{3}$ and $\mathbf{7}$ probably due to its slightly bulkier propyl groups from which solvation stabilization of $\mathbf{8}$ under the conditions suffers. An intermolecular charge-transfer interaction between the cations and dibenzo-24-crown-8 was also studied.
\end{abstract}

\section{Introduction}

We previously reported the synthesis and some crystal structures of various 1,1-spiroalkylated $1 H$-azulenium cations 1-5. ${ }^{1,2}$ Their $\mathrm{p} K_{\mathrm{R}}+$ values were in a range of 9.9-13.2, which are far greater than that of the tropylium cation. The order of the stability of the mono-spiroalkylated cations increases with the number of carbon atoms of the carbocycle at the 1-position, as 
$\mathbf{3}>\mathbf{2}>\mathbf{1}$ and the reluctance against electrochemical reduction showed a similar tendency. ${ }^{1}$ The enhanced thermodynamic stability is attributed to a hybrid stabilization of the inductive and $\sigma-\pi$ conjugation effects of the carbocycle and the $\pi-\pi$ conjugation of the double bond at the 2 and 3-positions. Since the degree of the $\pi-\pi$ conjugative effects is the same in a series of the cations, the order of the stability of mono-spiroalkylated cations should mainly meet the degree of the effects of the substituents at the 1-position; the cation having more carbon atoms in the substituents at the 1-position should be more stable. Herein we describe the synthesis of $1 H$-azulenium cations 6-8 substituted simply by linear alkyl groups and also their stability which does not come up to the order of the number of carbon atoms in the alkyl groups.

$<$ Insert Chart 1 and 2>

\section{Results and Discussion}

\subsection{Synthesis and physical properties of 1,1-dialkyl-1H-azulenium cations 6-8}

The title cations were synthesized by the method which we had developed for the spiroalkylated cation $\mathbf{3}^{3}$ (Scheme 1). The Mukaiyama aldol reaction ${ }^{4}$ of the trimethylsilyl enol ether 9 with the ketones in the presence of titanium tetrachloride and the subsequent Nazarov cyclization $^{5,6}$ provided the azulenones 13-15 in moderate yields. Since the yield of the keto-alcohol 12 was slightly less than those of others, the Noyori-Mukaiyama aldol reaction ${ }^{7}$ was also applied for 9 with 4,4-dimethoxyheptane. The reaction gave a mixture of two products, 22 and 23 (Scheme 2). Separation of the products by column chromatography resulted in isolation of only $\mathbf{2 3}$. So far, $\mathbf{2 2}$ has not been isolated, though its existence in the mixture was confirmed by its NMR spectrum. Without purification, this mixture was converted by the Nazarov cyclization into $\mathbf{1 5}$ in 78\% yield based on $\mathbf{9}$. The azulenones 13-15 were transformed into the 1,4-dihydroazulenes 19-21 via the tosylhydrazone 16-18 by the Shapiro reaction. ${ }^{8}$ The final hydride abstraction with trityl perchlorate $\left(\mathrm{Ph}_{3} \mathrm{C}^{+} \mathrm{ClO}_{4}{ }^{-}\right)$produced the desired cations 6-8. Their structures were fully characterized by spectroscopic and combustion analyses. The results of H-H COSY, HMQC and HMBC experiments covered the assignments of both proton and carbon signals indicated in the Experimental section except 
the carbon signals for the 6 and 7 positions of 6 . Their UV spectra showed three bands at 224-231, 264-271 and 358-363 nm as seen in the spectra of 1-3 (Table 1, Figures 1 and 2).

$<$ Insert Scheme 1 and 2, Table 1, and Figure 1 and 2>

\subsection{Thermodynamic stability and reduction potentials of 1,1-dialkyl-1H-azulenium cations}

Assuming equilibrium between a carbocation and its corresponding alcohol (Eq. (1)), the $\mathrm{p} K_{\mathrm{R}}+$ value can be determined, ${ }^{9}$ which indicates thermodynamic stability for the carbocation.

$$
\mathbf{R}^{+}+\mathrm{H}_{2} \mathbf{O} \rightleftharpoons \mathbf{R O H}+\mathbf{H}^{+}
$$

The $\mathrm{p} K_{\mathrm{R}}+$ values of 6-8 obtained by the UV method in $50 \%$ aqueous acetonitrile solutions were $8.6,10.1$ and 9.2, respectively (Table 2). The thermodynamic stability of these cations is comparable to those of the cations $\mathbf{1 - 3}$ and 1,2-bicyclo[2.2.2]octanotropyilum cation (24, $\left.\mathrm{p} K_{\mathrm{R}}+=8.8\right)^{10}$ and is greater than that $(3.88)^{11}$ of the tropylium cations. The order of the stability $(\mathbf{7}>\mathbf{8}>\mathbf{6})$ is not that expected from the number of carbon atoms in the alkyl groups. Since two propyl groups located at the 1-position of the cation $\mathbf{8}$ are slightly hydrophobic and slightly bulky to show steric hindrance, the solvation stabilization in the aqueous acetonitrile solution of the cation 8 must suffer. ${ }^{12}$ In other words, the degree of the inductive and conjugative effects of the propyl group in 8 can not be adequately evaluated from this $\mathrm{p} K_{\mathrm{R}}+$ value. On the other hand, tropylium ions in general show irreversible potentials by electrochemical reduction (Figure 3). The reduction potentials of tropylium ions indicate their reluctance for the reduction and provide another empirical stability parameter for carbocations. ${ }^{13}$ Reduction potentials for 6-8 measured by cyclic voltammetry in an acetonitrile solution with tetrabutylammonium perchlorate as a supporting electrolyte are shown in Table 2.

$<$ Insert Table 2, Chart 3 and Figure 3>

Although the potentials of $\mathbf{6 - 8}$ decrease with increasing number of carbon atoms in the 
substituents, the value of $\mathbf{8}$ is comparable to that of 7 , suggesting that stabilization by inductive and $\sigma-\pi$ conjugative effects of the propyl groups in $\mathbf{8}$ is the same as that of the ethyl groups in 7 . This is in contrast to the result in a case of 1,1 -spiroalkyl- $1 H$-azulenium cations 1-3. Specially, it is most clear when the cations with the same number of carbon atoms in the substituents at the 1-position between two series of 1,1-dialkyl- and 1,1-spiroalkyl cations are compared; 7 shows slightly greater stability than $\mathbf{1}$ based not only on the $\mathrm{p} K_{\mathrm{R}}+$ values but also on reduction potentials, though stability of $\mathbf{8}$ is less than that of. $\mathbf{3}$. Therefore, it is apparent that stabilization by inductive and $\sigma-\pi$ conjugative effects of the alkyl group in the 1,1-dialkyl system is almost saturated up to the number of six carbon atoms.

\section{Intermolecular charge-transfer interaction of the 1,1-substituted $1 \mathrm{H}$-azulenium cations with dibenzo-24-crown-8}

The tropylium cation as an acceptor shows intermolecular charge-transfer (ICT) interactions with $n$-donors of halide anions ${ }^{14}$ and $\pi$-donors of aromatic hydrocarbons. ${ }^{15}$ Benzene-substituted crown ethers are known to serve also as a $\pi$-donor and show ICT absorptions with tropylium cations. ${ }^{16}$ As a typical example, the tropylium cation and dibenzo-24-crown-8 yielded the 1:1 inclusion complex 25. ${ }^{17}$ We also are interested in whether the 1,1 -substituted $1 H$-azulenium cations interact with dibenzo-24-crown-8 or not. In order to examine such possibility, measurements of ${ }^{1} \mathrm{H}$ NMR and UV-Vis spectra in the presence of the crown ether were carried out. The difference between the average chemical shifts of the cationic protons in the presence and absence of the crown ether is quite small as shown in Table 3. The acetonitrile solutions containing a 1:1 mixture of the cation and the crown ether indicated clear ICT bands around 500 700 $\mathrm{nm}$. However, attempts to isolate inclusion complexes have met with little success so far. These results suggest that the interaction between the azulenium cations and the benzene ring of the crown ether occurs not in the way of the cationic part sandwiched between two benzene rings of the crown ether like $\mathbf{2 5}$ but the cationic part with one benzene ring at the outside of the crown molecule, probably due to larger molecular sizes of these azulenium cations than that of the tropylium cation. Further 
study for evaluating the associate constant for complexes and interaction using crown ethers with a larger core size is the focus of current investigations.

$<$ Insert Chart 4 and Table 3>

\section{Summary}

We have synthesized and characterized the 1,1-dialkyl-1H-azulenium cations 6-8 whose stability was discussed based on their $\mathrm{p} K_{\mathrm{R}}+$ values and reduction potentials. The $\mathrm{p} K_{\mathrm{R}}+$ value of the cation 8 is smaller relative to those of $\mathbf{1}, \mathbf{2}, \mathbf{3}$, and 7 . This can be ascribed to its restrained solvation stabilization due to its slightly bulkier substituents. Comparison of reduction potentials of various $1 H$-azulenium cations suggests that stabilization by inductive and $\sigma-\pi$ conjugative effects shows saturation up to the number of six carbon atoms in the alkyl group in the 1,1-dialkyl-1H-azulenium cations.

\section{Experimental Sections}

\subsection{General}

Melting points were measured on a Yanaco MP-3 and are uncorrected. IR spectra were recorded on a Perkin-Elmer Spectrum RX I spectrometer. UV spectra were measured on a Shimadzu UV-1600 spectrometer. ${ }^{1} \mathrm{H}$ and ${ }^{13} \mathrm{C}$-NMR were recorded with tetramethylsilane as an internal standard on JEOL $\alpha 400$ spectrometer. Mass spectra were measured on a JMS-700 mass spectrometer. Cyclic voltammograms were recorded on a Yanako P1100 instrument. Column chromatography was done with either Merck Kieselgel 60 Art 7734 or Wako activated alumina. Ether and THF were purified by distillation from sodium and benzophenone under argon atmosphere. An ether solution of methyl lithium was purchased from Kanto Chem. Co. and was titrated before use. Titanium tetrachloride was purchased from Wako Chem. and was used without purification. Dibenzo-24-crown-8 was purchased from Tokyo Kasei Industry. The syntheses of 1-(trimethylsiloxyvinyl)-1,3,5-cycloheptatriene 9 from 1-acetyl-1,3,5-cycloheptatriene and 3,3-dimethyl-1,2,3,8-tetrahydroazulenone $\mathbf{1 4}$ via 10 from 9 were previously reported. ${ }^{3}$ Trity perchrorate was prepared by the method of 
Dauben et al. ${ }^{18}$

\subsection{Synthesis of 3,3-diethyl- and 3,3-dipropyl-1,2,3,8-tetrahydroazulenones (14 and 15) by the Mukaiyama aldol method}

A solution of $2.06 \mathrm{~g}(10.0 \mathrm{mmol})$ of the silyl enol ether 9 in $30 \mathrm{ml}$ of dichloromethane was added dropwise to a solution of $1.30 \mathrm{ml}(11.0 \mathrm{mmol})$ of titanium chloride and $3.20 \mathrm{ml}(30.0$ mmol) of 3-pentanone in $20 \mathrm{ml}$ of dichloromethane at $0{ }^{\circ} \mathrm{C}$ under nitrogen atmosphere. After being stirred for $3 \mathrm{hr}$, the reaction mixture was poured into water. The aqueous layer was extracted with dichloromethane $(50 \mathrm{ml} \times 2)$. The combined organic layer was washed with a saturated $\mathrm{NaHCO}_{3}$ aqueous solution and brine, and then dried over anhydrous $\mathrm{MgSO}_{4}$. The solvent was removed under reduced pressure and the residue was purified by column chromatography $\left(\mathrm{SiO}_{2}\right.$, hexane-ethyl acetate (9:1) as eluent) to give $1.58 \mathrm{~g}$ (72\% yield) of $\mathbf{1 1}$ as a pale yellow oil. ${ }^{1} \mathrm{H}$ NMR $\left(\mathrm{CDCl}_{3}\right) \delta 0.86(\mathrm{t}, J=7.6 \mathrm{~Hz}, 6 \mathrm{H}), 1.53(\mathrm{~m}, 4 \mathrm{H}), 2.63(\mathrm{~d}, J=$ $7.1 \mathrm{~Hz}, 2 \mathrm{H}), 2.83(\mathrm{~s}, 2 \mathrm{H}), 4.15(\mathrm{br} \mathrm{s}, 1 \mathrm{H}), 5.58(\mathrm{dt}, J=9.4,7.1 \mathrm{~Hz}, 1 \mathrm{H}), 6.29(\mathrm{dd}, J=9.4,5.6$ $\mathrm{Hz}, 1 \mathrm{H}), 6.71(\mathrm{dd}, J=11.2,5.9 \mathrm{~Hz}, 1 \mathrm{H}), 6.88(\mathrm{dd}, J=11.2,5.9 \mathrm{~Hz}, 1 \mathrm{H}), 7.12(\mathrm{~d}, J=5.9 \mathrm{~Hz}$, 1H) ppm; ${ }^{13} \mathrm{C} \mathrm{NMR}\left(\mathrm{CDCl}_{3}\right)$ 8.1, 25.2, 31.3, 43.6, 74.4, 125.8, 127.3, 129.1, 131.9, 133.1, 136.5, $201.8 \mathrm{ppm}$; IR (KBr) $v_{\max }=1647 \mathrm{~s}, 732 \mathrm{~s} \mathrm{~cm}^{-1}$; MS $(20 \mathrm{eV}) \mathrm{m} / z$ (rel int) $220\left(\mathrm{M}^{+}, 2\right)$, 202 (18), 134 (18), 119 (93), 91 (100), 65 (25), 57 (28). Found: 220.1463. Calcd for $\mathrm{C}_{14} \mathrm{H}_{20} \mathrm{O}_{2}: \mathrm{M}, 220.1445$.

A mixture of $1.35 \mathrm{~g}(6.52 \mathrm{mmol})$ of $\mathbf{1 1}$ in formic acid $(7.5 \mathrm{ml})$ and phosphoric acid $(7.5 \mathrm{ml})$ was heated at $90{ }^{\circ} \mathrm{C}$ for $4 \mathrm{hr}$. Then the resulted dark brown mixture was poured into water and extracted with ether $(50 \mathrm{ml} \times 3)$. The combined organic layer was washed with a saturated $\mathrm{NaHCO}_{3}$ aqueous solution and brine, and then dried over anhydrous $\mathrm{MgSO}_{4}$. The solvent was removed under reduced pressure and the residue was purified by column chromatography $\left(\mathrm{SiO}_{2}\right.$, hexane-ethyl acetate (9:1) as eluent) to give $700 \mathrm{mg}$ (58\% yield) of $\mathbf{1 4}$ as a pale yellow oil. ${ }^{1} \mathrm{H}$ NMR $\left(\mathrm{CDCl}_{3}\right) \delta 0.68(\mathrm{t}, J=6.1 \mathrm{~Hz}, 6 \mathrm{H}), 1.61(\mathrm{~m}, 4 \mathrm{H}), 2.29(\mathrm{~s}, 2 \mathrm{H}), 2.68(\mathrm{~d}, J=6.5$ $\mathrm{Hz}, 2 \mathrm{H}), 5.65(\mathrm{dt}, J=9.9,6.5 \mathrm{~Hz}, 1 \mathrm{H}), 6.13(\mathrm{dd}, J=9.9,5.9 \mathrm{~Hz}, 1 \mathrm{H}), 6.63(\mathrm{~d}, J=11.3 \mathrm{~Hz}$, 1H), $6.80(\mathrm{dd}, J=11.3,5.9 \mathrm{~Hz}, 1 \mathrm{H}) \mathrm{ppm} ;{ }^{13} \mathrm{C} \mathrm{NMR}\left(\mathrm{CDCl}_{3}\right)$ 8.5, 21.5, 31.4, 44.3, 46.6, 125.4, 
127.2, 127.9, 133.5, 136.7, 168.5, 206.0 ppm; IR (KBr) $v_{\max }=2963 \mathrm{~s}, 1692 \mathrm{~s} \mathrm{~cm}^{-1}$; MS (70 eV) m/z (rel int) $202\left(\mathrm{M}^{+}, 80\right), 173$ (32), 145 (100), 131(39), 118 (58), 105 (22), 91 (45). Found: 202.1358. Calcd for $\mathrm{C}_{14} \mathrm{H}_{18} \mathrm{O}: \mathrm{M}, 202.1373$.

Similarly 15 was obtained via 12. 12; a pale yellow oil. ${ }^{1} \mathrm{H}$ NMR $\left(\mathrm{CDCl}_{3}\right) \delta 0.89(\mathrm{t}, J=7.2$ $\mathrm{Hz}, 6 \mathrm{H}), 1.23-1.52(\mathrm{~m}, 8 \mathrm{H}), 2.64(\mathrm{~d}, J=7.3 \mathrm{~Hz}, 2 \mathrm{H}), 2.83(\mathrm{~s}, 2 \mathrm{H}), 5.59$ (dt, $J=9.3,7.2 \mathrm{~Hz}$, 1H), $6.29(\mathrm{dd}, J=9.3,5.6 \mathrm{~Hz}, 1 \mathrm{H}), 6.70(\mathrm{dd}, J=11.2,6.0 \mathrm{~Hz}, 1 \mathrm{H}), 6.89$ (dd, $J=11.2,5.6 \mathrm{~Hz}$, 1H), $7.10(\mathrm{~d}, J=6.0 \mathrm{~Hz}, 1 \mathrm{H}) \mathrm{ppm} ;{ }^{13} \mathrm{C} \mathrm{NMR}\left(\mathrm{CDCl}_{3}\right)$ 14.6, 17.1, 25.2, 41.9, 44.4, 74.1, 125.8, 127.3, 129.0, 131.9, 133.0, 136.5, $201.8 \mathrm{ppm}$; IR (KBr) $v_{\max }=3480 \mathrm{brm}, 2933 \mathrm{~s}, 1650 \mathrm{~s} 1603 \mathrm{~s}$, $1408 \mathrm{~s}, 1384 \mathrm{~s}, 1200 \mathrm{~s}, 1170 \mathrm{~s}, 717 \mathrm{~s} \mathrm{~cm}^{-1}$; MS (20 eV) m/z (rel int) $230\left(\mathrm{M}^{+}-\mathrm{H}_{2} \mathrm{O} 19\right), 205$ (5), 134 (19), 133 (22), 119 (28), 118 (18), 115 (14), 105 (10), 103 (7), 92 (13), 91 (100), 90 (30), 89 (27). ${ }^{19}$ Found: 230.1636. Calcd for $\mathrm{C}_{16} \mathrm{H}_{24} \mathrm{O}_{2}-\mathrm{H}_{2} \mathrm{O}: \mathrm{M}, 230.1671$. 15; a pale yellow oil. ${ }^{1} \mathrm{H}$ $\operatorname{NMR}\left(\mathrm{CDCl}_{3}\right) \delta$ 0.83-0.91 (m, 2H), $0.85(\mathrm{t}, J=7.2 \mathrm{~Hz}, 6 \mathrm{H}), 1.09-1.19(\mathrm{~m}, 2 \mathrm{H}), 1.44(\mathrm{td}, J$ $=12.8,4.1 \mathrm{~Hz}, 2 \mathrm{H}), 1.57(\mathrm{td}, J=12.8,4.1 \mathrm{~Hz}, 2 \mathrm{H}), 2.32(\mathrm{~s}, 2 \mathrm{H}), 2.66(\mathrm{~d}, J=6.5 \mathrm{~Hz}, 2 \mathrm{H}), 5.65$ $(\mathrm{dt}, J=9.9,7.2 \mathrm{~Hz}, 1 \mathrm{H}), 6.13(\mathrm{dd}, J=9.9,6.0 \mathrm{~Hz}, 1 \mathrm{H}), 6.64(\mathrm{~d}, J=11.4 \mathrm{~Hz}, 1 \mathrm{H}), 6.78(\mathrm{dd}, J$ $=11.4,6.0 \mathrm{~Hz}, 1 \mathrm{H}) \mathrm{ppm} ;{ }^{13} \mathrm{C} \mathrm{NMR}\left(\mathrm{CDCl}_{3}\right)$ 14.6, 17.7, 21.6, 41.7, 45.7, 46.1, 125.6, 127.4, 128.1, 133.0, 136.8, 169.4, 206.4 ppm; IR $(\mathrm{KBr}) v_{\max }=2957 \mathrm{~s}, 2929 \mathrm{~s}, 1692 \mathrm{~s} 1272 \mathrm{~s} \mathrm{~cm}^{-1}$; MS $(70 \mathrm{eV}) \mathrm{m} / z$ (rel int) $231\left(\mathrm{M}^{+}+1,19\right), 230\left(\mathrm{M}^{+}, 100\right), 224$ (21), 188 (31), 187 (40), 167 (15), 159 (81), 149 (50), 145 (84). Found: 230.1674. Calcd for $\mathrm{C}_{16} \mathrm{H}_{22} \mathrm{O}: \mathrm{M}, 230.1671$.

\subsection{Synthesis of 3,3-dipropyl-1,2,3,8-tetrahydroazulenone (21) by the Noyori-Mukaiyama aldol method}

To a solution of $5.15 \mathrm{~g}(25.0 \mathrm{mmol})$ of the silyl enol ether 9 and $4.41 \mathrm{~g}(27.5 \mathrm{mmol})$ of 4,4-dimethoxyheptane in $125 \mathrm{ml}$ of dichloromethane at $-78{ }^{\circ} \mathrm{C}$ under nitrogen atmosphere was added $0.45 \mathrm{ml}(2.5 \mathrm{mmol})$ of trimethylsilyl triflate. After being stirred at the same temperature for $19 \mathrm{hr}$, the reaction mixture was poured into water. The aqueous layer was extracted with dichloromethane $(50 \mathrm{ml} \times 2)$. The combined organic layer was washed with a saturated $\mathrm{NaHCO}_{3}$ aqueous solution and brine, and then dried over anhydrous $\mathrm{MgSO}_{4}$. The solvent was removed under reduced pressure and the residue was purified by column 
chromatography $\left(\mathrm{SiO}_{2}\right.$, hexane-ethyl acetate $(96: 4)$ as eluent) to give $5.98 \mathrm{~g}$ of a mixture of 22 and $\mathbf{2 3}$ as a pale yellow oil. This mixture was dissolved in formic acid (40 $\mathrm{ml})$ and phosphoric acid $(40 \mathrm{ml})$ and was heated at $90{ }^{\circ} \mathrm{C}$ for $4 \mathrm{hr}$. The resulted dark brown mixture was poured into ice-water $(100 \mathrm{ml})$ and extracted with ether $(100 \mathrm{ml} \mathrm{x} \mathrm{3)}$. The combined organic layer was washed with a saturated $\mathrm{NaHCO}_{3}$ aqueous solution and brine, and then dried over anhydrous $\mathrm{MgSO}_{4}$. The solvent was removed under reduced pressure and the residue was purified by column chromatography $\left(\mathrm{SiO}_{2}\right.$, hexane-ethyl acetate $(9: 1)$ as eluent) to give $4.48 \mathrm{~g}$ (78\% yield based on $\mathbf{9}$ ) of $\mathbf{1 5}$ as a pale yellow oil.

Purification of a mixture of $\mathbf{2 2}$ and $\mathbf{2 3}$ by column chromatography gave $\mathbf{2 3}$ as a pale yellow oil. ${ }^{1} \mathrm{H}$ NMR $\left(\mathrm{CDCl}_{3}\right) \delta 0.94(\mathrm{t}, J=7.3 \mathrm{~Hz}, 6 \mathrm{H}), 1.45-1.55(\mathrm{~m}, 4 \mathrm{H}), 2.14(\mathrm{t}, J=7.6 \mathrm{~Hz}, 2 \mathrm{H})$, $2.42(\mathrm{t}, J=7.9 \mathrm{~Hz}, 2 \mathrm{H}), 2.67(\mathrm{~d}, J=7.2 \mathrm{~Hz}, 2 \mathrm{H}), 5.59$ (dt, $J=8.9,7.2 \mathrm{~Hz}, 1 \mathrm{H}), 6.26$ (dd, $J=$ 9.3, $5.6 \mathrm{~Hz}, 1 \mathrm{H}), 6.41(\mathrm{~s}, 1 \mathrm{H}), 6.68(\mathrm{dd}, J=11.1,6.0 \mathrm{~Hz}, 1 \mathrm{H}), 6.81(\mathrm{dd}, J=11.1,5.6 \mathrm{~Hz}, 1 \mathrm{H})$, $7.05(\mathrm{~d}, J=6.0 \mathrm{~Hz}, 1 \mathrm{H}) \mathrm{ppm} ;{ }^{13} \mathrm{C} \mathrm{NMR}\left(\mathrm{CDCl}_{3}\right)$ 13.9, 14.4, 21.0, 21.9, 25.9, 34.7, 40.5, $120.8,125.4,127.0,129.3,131.3,139.9,135.2,161.4,191.5$ ppm; UV $\left(\mathrm{CH}_{3} \mathrm{OH}\right) \lambda_{\max }=291$ $(\log \varepsilon=3.91), 318 \mathrm{sh}(3.78) \mathrm{nm} ; \mathrm{IR}(\mathrm{KBr}) v_{\max }=2957 \mathrm{~s}, 2929 \mathrm{~s}, 1692 \mathrm{~s} 1272 \mathrm{~s} \mathrm{~cm}^{-1} ; \mathrm{MS}(70 \mathrm{eV})$ m/z (rel int) $230\left(\mathrm{M}^{+}, 100\right), 201$ (29), 187 (45), 159 (22), 145 (78), 139 (48), 119 (26), 91 (78). Found: 230.1670 . Calcd for $\mathrm{C}_{16} \mathrm{H}_{22} \mathrm{O}$ : M, 230.1671.

\subsection{Synthesis of 1,1-dimethyl-, 1,1-diethyl-, and 1,1-dipropyl-1,4-dihydroazulenes (19 20, and 21)}

To a suspension of $14(0.841 \mathrm{~g}, 4.43 \mathrm{mmol})$ and $0.824 \mathrm{~g}(4.43 \mathrm{mmol})$ tosylhydrazide in $5 \mathrm{ml}$ of dry THF was stirred at $40^{\circ} \mathrm{C}$ for $72 \mathrm{hr}$. The solids formed were collected by filteration and washed well with ether to give $0.848 \mathrm{~g}$ of 16 (56\% yield) as a white powder. Analytical samples of 16 were obtained by recrystallization from hexane-dichloromethane.

16; colorless microcrystals, mp 197-198 ${ }^{\circ} \mathrm{C}$. Found: C, 66.39; H, 6.48; N, 8.25\%. Calcd for $\mathrm{C}_{19} \mathrm{H}_{22} \mathrm{~N}_{2} \mathrm{O}_{2} \mathrm{~S}: \mathrm{C}, 66.64 ; \mathrm{H}, 6.47 ; \mathrm{N}, 8.18 \%$.

To a suspension of $1.00 \mathrm{~g}(2.92 \mathrm{mmol})$ of $\mathbf{1 6}$ in $20 \mathrm{ml}$ of dry ether at $0{ }^{\circ} \mathrm{C}$ under nitrogen atmosphere was added $50 \mathrm{ml}$ of methyl lithium solution ( $0.8 \mathrm{M}$ ether solution, $40 \mathrm{mmol})$ 
slowly through a syringe. After the addition, the mixture was stirred for $12 \mathrm{hr}$. The mixture was quenched by adding water carefully and was poured into a mixture of ether and ice-water. The aqueous layer was extracted with ether $(50 \mathrm{ml} \mathrm{x} \mathrm{2).} \mathrm{The} \mathrm{combined} \mathrm{organic} \mathrm{layer} \mathrm{was}$ washed with brine and dried over anhydrous $\mathrm{MgSO}_{4}$. The solvent was removed under reduced pressure and the residue was purified by column chromatography $\left(\mathrm{SiO}_{2}\right.$, hexane as eluent) to give $273 \mathrm{mg}$ (59\% yield) of 19 as a pale yellow oil. ${ }^{1} \mathrm{H}$ NMR $\left(\mathrm{CDCl}_{3}\right) \delta 1.14(\mathrm{~s}, 6 \mathrm{H}), 2.69(\mathrm{~d}$, $J=6.4 \mathrm{~Hz}, 2 \mathrm{H}), 5.35(\mathrm{dt}, J=9.6,6.8 \mathrm{~Hz}, 1 \mathrm{H}), 6.08(\mathrm{dd}, J=9.6,6.0 \mathrm{~Hz}, 1 \mathrm{H}), 6.08(\mathrm{~d}, J=5.6$ $\mathrm{Hz}, 1 \mathrm{H}), 6.37$ (d, $J=5.6 \mathrm{~Hz}, 1 \mathrm{H}), 6.39$ (dd, $J=10.8,6.0 \mathrm{~Hz}, 1 \mathrm{H}), 6.56(\mathrm{~d}, J=10.8 \mathrm{~Hz}, 1 \mathrm{H})$ ppm; ${ }^{13} \mathrm{C}$ NMR $\left(\mathrm{CDCl}_{3}\right) \delta 22.6,27.9,51.8,120.7,125.2,127.37,127.40,130.6,132.8,148.6$, $149.2 \mathrm{ppm}$; IR $(\mathrm{KBr}) v_{\max }=2925 \mathrm{~s}, 732 \mathrm{~s} \mathrm{~cm}^{-1}$; MS $(70 \mathrm{eV}) \mathrm{m} / z$ (rel int) $158\left(\mathrm{M}^{+}, 35\right), 143$ (100), 128 (74), 115 (18), 91 (23), 77 (21), 58 (22). Found: 158.1049. Calcd for $\mathrm{C}_{12} \mathrm{H}_{14}$ : M, 158.0960.

Similarly, $\mathbf{1 4}$ and $\mathbf{1 5}$ were transformed to $\mathbf{2 0}$ without isolating $\mathbf{1 7}$ in $31 \%$ based on $\mathbf{1 4}$ and to 21.

20; a pale yellow oil. ${ }^{1} \mathrm{H}$ NMR $\left(\mathrm{CDCl}_{3}\right) \delta 0.5 !(\mathrm{t}, J=7.3 \mathrm{~Hz}, 6 \mathrm{H}), 1.52(\mathrm{dq}, J=11.4,7.3 \mathrm{~Hz}$, 2H), $1.73(\mathrm{dq}, J=11.4,7.3 \mathrm{~Hz}, 2 \mathrm{H}), 2.68(\mathrm{~d}, J=6.6 \mathrm{~Hz}, 2 \mathrm{H}), 5.34(\mathrm{dt}, J=9.6,6.6 \mathrm{~Hz}, 1 \mathrm{H})$, $6.06(\mathrm{dd}, J=9.8,5.9 \mathrm{~Hz}, 1 \mathrm{H}), 6.17(\mathrm{~d}, J=5.4 \mathrm{~Hz}, 1 \mathrm{H}), 6.22(\mathrm{~d}, J=5.4 \mathrm{~Hz}, 1 \mathrm{H}), 6.38(\mathrm{dd}, J=$ 11.0, 5.6 Hz, $1 \mathrm{H}), 6.46(\mathrm{~d}, J=11.0 \mathrm{~Hz}, 1 \mathrm{H}) \mathrm{ppm} ;{ }^{13} \mathrm{C} \mathrm{NMR}\left(\mathrm{CDCl}_{3}\right) \delta 8.7,27.8,29.0,60.4$, $120.4,125.5,127.1,128.7,133.0,135.0,144.9,145.4$ ppm; IR $(\mathrm{KBr}) v_{\max }=2964 \mathrm{~s}, 2931 \mathrm{~s}$, 729 cm $\mathrm{s} \mathrm{c}^{-1}$; MS (70 eV) m/z (rel int) $186\left(\mathrm{M}^{+}, 19\right), 171$ (27), 157 (69), 141 (55), 129 (100), 115 (43), 105 (24), 91 (46), 77 (23), 57 (63). Found: 186.1437. Calcd for $\mathrm{C}_{14} \mathrm{H}_{18}$ : M, 186.1409.

18; creamy white solids, mp $139-141{ }^{\circ} \mathrm{C}$. Found: C, 69.32; H, 7.71; N, 6.92\%. Calcd for $\mathrm{C}_{23} \mathrm{H}_{30} \mathrm{~N}_{2} \mathrm{O}_{2} \mathrm{~S}: \mathrm{C}, 69.31 ; \mathrm{H}, 7.59 ; \mathrm{N}, 7.03 \%$.

21; a pale yellow oil, ${ }^{1} \mathrm{H}$ NMR $\left(\mathrm{CDCl}_{3}\right) \delta 0.75(\mathrm{t}, J=6.8 \mathrm{~Hz}, 6 \mathrm{H}), 0.78-0.89(\mathrm{~m}, 2 \mathrm{H})$, 0.90-1.05 (m, 2H), 1.44 (m, 2H), $1.66(\mathrm{~m}, 2 \mathrm{H}), 2.67$ (d, $J=6.4 \mathrm{~Hz}, 2 \mathrm{H}), 5.33$ (dt, $J=9.8,6.4$ $\mathrm{Hz}, 1 \mathrm{H}), 6.06(\mathrm{dd}, J=9.8,6.0 \mathrm{~Hz}, 1 \mathrm{H}), 6.13(\mathrm{~d}, J=5.4 \mathrm{~Hz}, 1 \mathrm{H}), 6.27(\mathrm{~d}, J=5.4 \mathrm{~Hz}, 1 \mathrm{H})$, $6.36(\mathrm{dd}, J=11.0,6.0 \mathrm{~Hz}, 1 \mathrm{H}), 6.49(\mathrm{~d}, J=11.0 \mathrm{~Hz}, 1 \mathrm{H}) \mathrm{ppm} ;{ }^{13} \mathrm{C} \mathrm{NMR}\left(\mathrm{CDCl}_{3}\right)$ 14.7, 17.4, $27.8,38.7,59.8,120.4,125.6,127.1,127.2,132.4,134.5,145.8,146.3$ ppm; IR $(\mathrm{KBr}) v_{\max }=$ 
2955s, 2929s, 2870s, 731s cm ${ }^{-1}$; MS (70 eV) m/z (rel int) $214\left(\mathrm{M}^{+}, 32\right), 213(53), 185(32)$, 171 (39), 155 (31), 143 (49), 142 (32), 131 (21), 129 (100), 128 (60),117 (36), 115 (49). Found: 214.1650. Calcd for $\mathrm{C}_{16} \mathrm{H}_{22}$ : M, 214.1721.

\subsection{Synthesis of 1,1-dimethyl-, 1,1-diethyl-, and 1,1-dipropyl-1H-azulenium perchlorates $(6,7,8)$}

To a solution of the precursor hydrocarbon $(1.00 \mathrm{mmol})$ in $10 \mathrm{ml}$ of acetonitrile at $0{ }^{\circ} \mathrm{C}$ under nitrogen atmosphere was added trityl perchlorate $(1.00 \mathrm{mmol})$ in one portion. The mixture was stirred at room temperature for $1 \mathrm{hr}$. The solvent was removed under reduced pressure and the residue was dissolved in the least amount of dichloromethane. Ether was added to the solution and solids formed were collected and washed well with cold ether.

6; yellow microcrystals, mp 99-100 ${ }^{\circ} \mathrm{C} .{ }^{1} \mathrm{H}$ NMR $\left(\mathrm{CD}_{3} \mathrm{CN}\right) \tilde{\delta} \tilde{1} \quad(\mathrm{~s}, \mathrm{Me}), 7.37$ (d, $J=5.2$ Hz, H-3), 7.73 (d, $J=5.2 \mathrm{~Hz}, \mathrm{H}-2), 8.76$ (tt, $J=9.7,1.4 \mathrm{~Hz}, \mathrm{H}-6), 8.83$ (td, $J=9.7,1.4 \mathrm{~Hz}$, H-7), 8.91 (td, $J=9.7,1.4 \mathrm{~Hz}, \mathrm{H}-5), 9.00$ (dd, $J=9.7,1.4 \mathrm{~Hz}, \mathrm{H}-4), 9.02$ (dd, $J=9.7,1.4 \mathrm{~Hz}$, $\mathrm{H}-8)$ ppm; ${ }^{13} \mathrm{C}$ NMR $\left(\mathrm{CD}_{3} \mathrm{CN}\right) \delta 21.6(\mathrm{Me}), 57.8$ (C-1), 133.4 (C-3), $145.6(\mathrm{C}-4), 146.1$ (C-8), 149.7 (C-6 or 7), 149.8 (C-7 or 6), 153.1 (C-5), 167.8 (C-2), 171.0 (C-3a), 180.0 (C-8a) ppm; IR (KBr) $v_{\max }=1087 \mathrm{~s}, 626 \mathrm{~s} \mathrm{~cm}^{-1}$; MS (70 eV) m/z (rel int) $157\left(\mathrm{C}_{12} \mathrm{H}_{13}{ }^{+}, 17\right), 156(68), 141$ (100), 128 (23), 115 (28). Found: C, 56.20; H, 5.18\%. Calcd for $\mathrm{C}_{12} \mathrm{H}_{13} \mathrm{ClO}_{4}$ : C, 56.15; H, $5.10 \%$.

7; faintly greenish microcrystals, mp 97-99 ${ }^{\circ} \mathrm{C} .{ }^{1} \mathrm{H} \mathrm{NMR}\left(\mathrm{CD}_{3} \mathrm{CN}\right) \delta 0.50(\mathrm{t}, J=7.4 \mathrm{~Hz}, \mathrm{Me})$, $2.17\left(\mathrm{dq}, J=14.4,7.4 \mathrm{~Hz}\right.$, one of $\left.\mathrm{CH}_{2}\right), 2.32\left(\mathrm{dq}, J=14.4,7.4 \mathrm{~Hz}\right.$, one of $\left.\mathrm{CH}_{2}\right), 7.52(\mathrm{~d}, J=$ $5.6 \mathrm{~Hz}, \mathrm{H}-3), 7.71$ (d, $J=5.2 \mathrm{~Hz}, \mathrm{H}-2), 8.80$ (tt, $J=10.0,1.3 \mathrm{~Hz}, \mathrm{H}-6), 8.85$ (td, $J=10.0,1.3$ Hz, H-5), 8.95 (td, $J=10.0,1.3 \mathrm{~Hz}, \mathrm{H}-5), 8.99$ (dd, $J=10.0,1.3 \mathrm{~Hz}, \mathrm{H}-4), 9.02$ (dd, $J=10.0$, $1.3 \mathrm{~Hz}, \mathrm{H}-8) \mathrm{ppm} ;{ }^{13} \mathrm{C}$ NMR $\left(\mathrm{CD}_{3} \mathrm{CN}\right) \delta 9.0(\mathrm{Me}), 29.7\left(\mathrm{CH}_{2}\right), 67.3(\mathrm{C}-1), 136.0(\mathrm{C}-3), 144.6$ (C-4), 145.3 (C-8), 149.5 (C-7), 149.7 (C-6), 153.0 (C-5), 165.7 (C-2), 172.5 (C-3a), 178.5 (C-8a) ppm; IR (KBr) $v_{\max }=1449 \mathrm{~s}, 1095 \mathrm{~s}, 623 \mathrm{~s} \mathrm{~cm}^{-1}$; MS $(70 \mathrm{eV}) \mathrm{m} / \mathrm{z}$ (rel int) $185\left(\mathrm{C}_{14} \mathrm{H}_{17}{ }^{+}\right.$, 88), 171 (21), 169 (30), 157 (78), 141 (100), 129 (95), 128 (56), 115 (41). Found: C, 58.88; H, 5.97\%. Calcd for $\mathrm{C}_{12} \mathrm{H}_{13} \mathrm{ClO}_{4}$ : C, 59.06; H, 6.02\%. 
8; faintly brownish microcrystals, mp $148-150{ }^{\circ} \mathrm{C} .{ }^{1} \mathrm{H}$ NMR $\left(\mathrm{CD}_{3} \mathrm{CN}\right) \delta$ 0.55-0.66 (m, one of $\mathrm{H}-2$ ', 2H), 0.73 (t, $J=7.1 \mathrm{~Hz}, \mathrm{Me}, 6 \mathrm{H}), 0.96-1.07$ (m, one of H-2', 2H), 2.07 (m, one of H-1', 2H), 2.32 (m, one of H-1', 2H), 7.45 (d, $J=5.4 \mathrm{~Hz}, \mathrm{H}-3), 7.73$ (d, $J=5.4 \mathrm{~Hz}, \mathrm{H}-2), 8.75$ (tt, $J$ $=10.0,1.5 \mathrm{~Hz}, \mathrm{H}-6), 8.79(\mathrm{td}, J=10.0,1.5 \mathrm{~Hz}, \mathrm{H}-5), 8.90(\mathrm{td}, J=10.0,1.5 \mathrm{~Hz}, \mathrm{H}-5), 8.96$ (d-like, H-4 \& H-8) ppm; ${ }^{13} \mathrm{C}$ NMR $\left(\mathrm{CD}_{3} \mathrm{CN}\right) \delta 14.4$ (C-3', Me), $18.3\left(\mathrm{C}-2\right.$ ', $\left.\mathrm{CH}_{2}\right), 38.9$ (C-1', $\mathrm{CH}_{2}$ ), 66.5 (C-1), 135.3 (C-3), 144.5 (C-4), 145.2 (C-8), 149.3 (C-7), 149.5 (C-6), 152.7 (C-5), 166.1 (C-2), 172.1 (C-3a), 178.9 (C-8a) ppm; IR (KBr) $v_{\max }=1490 \mathrm{~s}, 1450 \mathrm{~s}, 1095 \mathrm{~s}, 831 \mathrm{~s}$, $623 \mathrm{~s} \mathrm{~cm}^{-1}$; MS (70 eV) m/z (rel int) $213\left(\mathrm{C}_{16} \mathrm{H}_{21}{ }^{+}, 4\right), 212$ (21), 184 (16), 183 (100), 165 (16), 155 (16), 154 (28), 153 (21), 152 (20), 141 (13), 128 (10). Found: C, 61.86; H, 6.81\%. Calcd for $\mathrm{C}_{16} \mathrm{H}_{21} \mathrm{ClO}_{4}$ : C, 61.44; $\mathrm{H}, 6.77 \%$.

\subsection{Determination of $\mathrm{p} K_{\mathrm{R}}+$ values}

The UV spectra in various $\mathrm{pH}$ of $50 \%$ aqueous acetonitrile solutions were measured by exactly the same method of Komatsu et al. ${ }^{11}$ Observed absorbance at the longest absorption maxima at $358-363 \mathrm{~nm}$ was ploted against $\mathrm{pH}$ to give a classical titration curve, whose midpoint was taken as the $\mathrm{p} K_{\mathrm{R}}+$.

\subsection{Cyclic voltammetry}

A standard three-electrode cell configuration was employed using a glassy carbon disk working electrode, a $\mathrm{Pt}$ wire auxiliary electrode, and an $\mathrm{Ag}$ wire as an $\mathrm{Ag} / \mathrm{Ag}+$ quasi-reference electrode. The reference electrode was calibrated at the completion of each measurement on a saturated calomel electrode (SCE). Cyclic voltammetry was measured in an acetonitrile solution with tetrabutylammonium perchlorate as a supporting electrolyte and a scan rate of $0.1 \mathrm{Vs}^{-1}$ at $25{ }^{\circ} \mathrm{C}$. Under this conditions, ferrocene showed a half wave oxidation potential of $+0.40 \mathrm{~V}$ vs SCE.

\section{Acknowledgement}

We gratefully thank Mr. Takuya Uchiyama and Mr. Hitoshi Kainuma at University of 
Toyama for their preliminary synthetic efforts.

\section{References and Notes}

1. Oda, M.; Sakamoto, A.; Uchiyama, T.; Kajioka, T.; Miyatake, R.; Kuroda, S. Tetrahedron Lett. 1999, 40, 3595-3596; Oda, M.; Fukuta, A.; Kajioka, T.; Uchiyama, T.; Miyatake, R.; Kuroda, S. Tetrahedron 2000, 56, 9917-9925; Oda, M.; Fukuta, A.; Uchiyama, T.; Kajioka, T.; Kuroda, S. Recent Research Developments in Organic Chemistry Vol. 6; Transworld Research Network, Trivandrum, 2002, Vol. 6, 543-563. For our related carbocations with smaller rings, see; Oda, M.; Kajioka, T.; Okujima, T.; Itoh S.; Morita, N.; Miyatake, R.; Kuroda, S. Chem. Lett. 1997, 1011-1012; Oda, M.; Kajioka, T.; Okujima, T.; Uchiyama, T.; Nagara, K.; Itoh S.; Morita, N.; Sato, T.; Miyatake, R.; Kuroda, S. Tetrahedron 1999, 55, 6081-6096; Oda, M.; Kajioka, T.; Kawamori, Y.; Uchiyama, T.; Kuroda, S.; Morita, N. Recent Research Developments in Organic \& Bioorganic Chemistry, Vol. 4; Transworld Research Network, Trivandrum, 2001, Vol. 4, pp133-149; Oda, M.; Sakamoto, A.; Miyatake, R.; Kuroda, S. Tetrahedron Lett. 1998, 39, 6195-6198; Oda, M.; Sakamoto, A.; Kajioka, T.; Miyatake, R.; Kuroda, S. Tetrahedron 1999, 55, 12479-12492.

2. Oda, M.; Kainuma, H.; Miyatake, R.; Kuroda, S. Tetrahedron Lett. 2002, 43, 3485-3488; Oda, M.; Kainuma, H.; Uchiyama, T.; Miyatake, R.; Kuroda, S. Tetrahedron 2003, 59, 2831-2841.

3. Oda, M.; Kajioka, T.; Ikeshima, K.; Miyatake, R.; Kuroda, S. Synth. Commun.. 2000, 30, 2335-2343. We had already reported the synthesis of the azulenone $\mathbf{1 3}$ from 9.

4. Mukaiyama, K.; Narasaka, K. Org. Synth. 1987, 65, 6-11.

5. For recent our results for preparing tetrahydroazulen-1-ones by the Nazarov cyclization, see; Oda, M.; Yamazaki, T.; Kajioka, T.; Miyatake, R.; Kuroda, S. Liebigs Ann./Recueil 1997, 2563-2566; Kajioka, T.; Oda, M.; Yamada, S.; Miyatake, R.; Kuroda, S. Synthesis 
1999, 184-187; Oda, M.; Kajioka, T.; Haramoto, K.; Miyatake, R.; Kuroda, S. Synthesis 1999, 1349-1353.

6. For recent reviews of the Nazarov cyclizations, see; Tius, M. A. Eur. J. Org. Chem. 2005, 2193-2206; Pellissier, H. Tetrahedron 2005, 61, 6479-6517.

7. $\quad$ Murata, S.; Suzuki, M.; Noyori, R. Tetrahedron Lett. 1980, 21, 2527-2528.

8. Shapiro, R. H. Org. React. 1976, 23, 405-507.

9. Doering, W. v. E.; Knox, L. H. J. Am. Chem. Soc. 1954, 76, 3203-3206; Doering, W. v. E.; Knox, L. H. J. Am. Chem. Soc. 1957, 79, 3203-3206.

10. Nakazawa, T.; Niimoto, Y.; Kubo, K.; Murata, I. Angew. Chem. 1980, 92, 566; Angew. Chem., Int. Ed. Engl. 1980, 19, 545-546.

11. Komatsu, K.; Akamatsu, H. ; Jinbu, Y.; Okamoto, K. J. Am. Chem. Soc. 1988, 110, 633-634; Komatsu, K.; Akamatsu, H.; Aonuma, S.; Jinbu, Y.; Maekawa, N.; Takeuchi, K. Tetrahedron 1991, 47, 6951-6966.

12. For clusters of solvated tropyium cations, see; Kinoshita, T.; Wakisaka, A.; Yasumoto, C. Takeuchi, K.; Yoshizawa, K.; Suzuki, A.; Yamabe, T. Chem. Commun. 2001, 1768-1769.

13. For linear relationships between thermodynamic and kinetic parameters of aromatic carbocations, see; Okamoto, K.; Takeuchi, K.; Kouichi, K.; Kubota, Y.; Ohara, R.; Arima, M.; Takahashi, K.; Waki, Y.; Shirai, S. Tetrahedron 1983, 39, 4011-4024.

14. Harmon, K. M.; Cummings, F. E.; Davis, D. A.; Diestler, D. J. Am. Chem. Soc. 1962, 84, $3349-3355$.

15. Feldman, M.; Winstein, S. J. Am. Chem. Soc. 1961, 83, 3338-3339.

16. Lämsä, M.; Kuokkanen, T.; Jalonen, J.; Virtanen, O. J. Phys. Org. Chem. 1995, 8, 377-384; Lämsä, M.; Kuokkanen, T. J. Phys. Org. Chem. 1996, 9, 21-28.

17. Lämsä, M.; Suorsa, T.; Pursiainen, J.; Kuokkanen, T.; Rissanen, K. Chem. Commun. 1996, $1443-1445$.

18. Dauben, Jr. H. J.; Lewis, R.; Honnen, R.; Harman, K. M. J. Org. Chem. 1960, 25, $1442-1445$. 
19. No molecular ion peak was observed even with low ionization energy of $20 \mathrm{eV}$ presumably because elimination of water should benefit from relief of steric strain. 
Table 1. UV absorption maxima in acetonitrile of $\mathbf{1 , 2}$, 3, 6, 7, and 8

\begin{tabular}{cc}
\hline Compounds & $\lambda_{\max }$ in nm $(\log \varepsilon)$ \\
\hline $\mathbf{1}$ & $230(4.31), 272(4.44), 364(4.00)$ \\
$\mathbf{2}$ & $230(4.26), 272(4.33), 367(4.07)$ \\
$\mathbf{3}$ & $227(4.29), 268(4.34), 362(4.03)$ \\
$\mathbf{6}$ & $222(4.33), 264(4.43), 358(4.09)$ \\
$\mathbf{7}$ & $228(4.15), 268(4.18), 362(3.94)$ \\
$\mathbf{8}$ & $231(4.29), 271(4.35), 363(4.08)$ \\
\hline
\end{tabular}


Table 2. The $\mathrm{p} K_{\mathrm{R}}+$ values and reduction potentials of $\mathbf{1 , 2 , 3}$, $\mathbf{6 , 7}$, and $\mathbf{8}$

\begin{tabular}{ccc}
\hline Compounds & $\mathrm{pK}_{\mathrm{R}}+$ value $^{\mathrm{a}}$ & $\begin{array}{c}\text { reduction potentials } \\
(\mathrm{V} \text { vs SCE })^{\mathrm{b}}\end{array}$ \\
\hline $\mathbf{1}$ & 9.9 & -0.41 \\
$\mathbf{2}$ & 10.0 & -0.46 \\
$\mathbf{3}$ & 10.4 & -0.54 \\
$\mathbf{6}$ & 8.6 & -0.38 \\
$\mathbf{7}$ & 10.1 & -0.45 \\
$\mathbf{8}$ & 9.2 & -0.46 \\
\hline
\end{tabular}

a)For measurements, see experiments.b)Irreversible. 
Table 3. The change of average chemical shifts of the cations $\mathbf{1}, \mathbf{2}, \mathbf{3}, \mathbf{6}, \mathbf{7}$, and $\mathbf{8}$ in the presence of dibenzo-24-crown-8 and ICT absorption bands

\begin{tabular}{ccc}
\hline Compounds & $\begin{array}{c}\text { Change of the } \\
\text { shift }^{\mathrm{a})}(\delta \mathrm{ppm})\end{array}$ & $\begin{array}{c}\text { ICT absorption wave- } \\
\text { length (nm) }\end{array}$ \\
\hline $\mathbf{1}$ & +0.031 & 599,646 \\
$\mathbf{2}$ & +0.025 & $650,715 \mathrm{sh}$ \\
$\mathbf{3}$ & +0.016 & 554,667 \\
$\mathbf{6}$ & +0.04 & 655 \\
$\mathbf{7}$ & +0.01 & $520 \mathrm{sh}$ \\
$\mathbf{8}$ & +0.006 & $509 \mathrm{sh}$ \\
tropylium & & \\
ion & +0.11 & $425^{\mathrm{c})}$ \\
\hline
\end{tabular}

a)The average shifts of the seven-membered ring protons of a 1:1 mixture in $\mathrm{CDCl}_{3}$ solutions.b)Measured in acetonitrile. c) Taken from ref 16. 


\section{Chart 1}

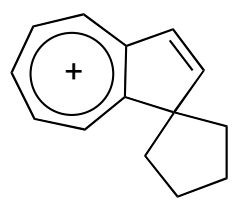

1

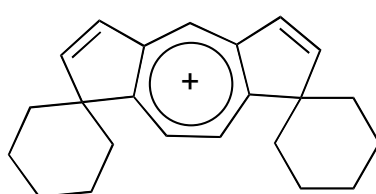

4

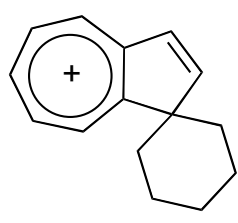

2

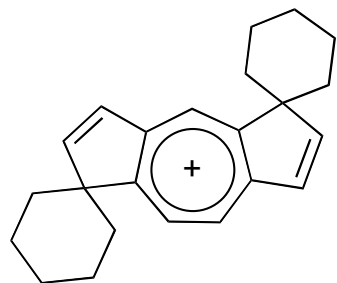

5

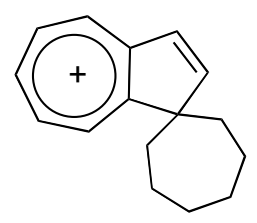

3

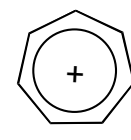

Tropylium ion 


\section{Chart 2}

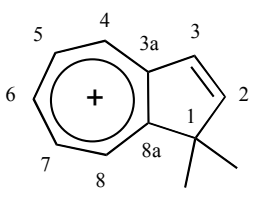

6

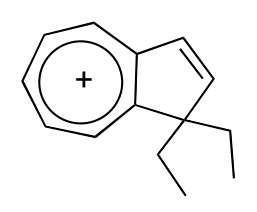

7

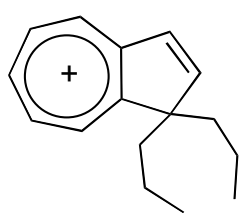

8 


\section{Chart 3}

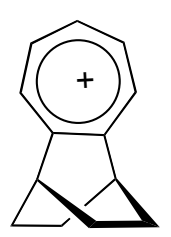

24

\section{Chart 4}

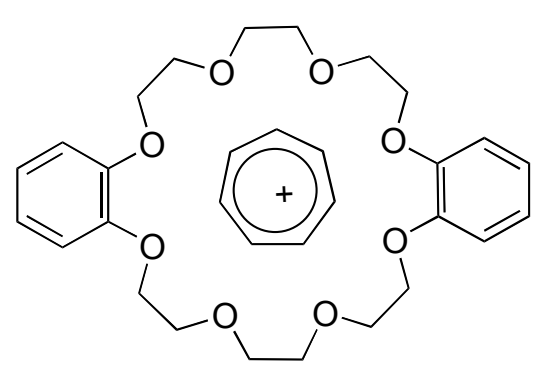

25 
Scheme 1<smiles>[R]C(=O)O[Sb](C)(=O)OC(=C)C1=CC=CC=CC1</smiles>

9 $10(\mathrm{R}=\mathrm{Me}, 59 \%)$<smiles>[R]C1([R])CC(=O)C2=C1C=CC=CC2</smiles>

11 ( $R=E t, 72 \%)$ $12(\mathrm{R}=\mathrm{Pr}, 41 \%)$

13 ( $R=M e, 68 \%)$

$14(\mathrm{R}=\mathrm{Et}, 58 \%)$

$15(\mathrm{R}=\mathrm{Pr}, 69 \%)$

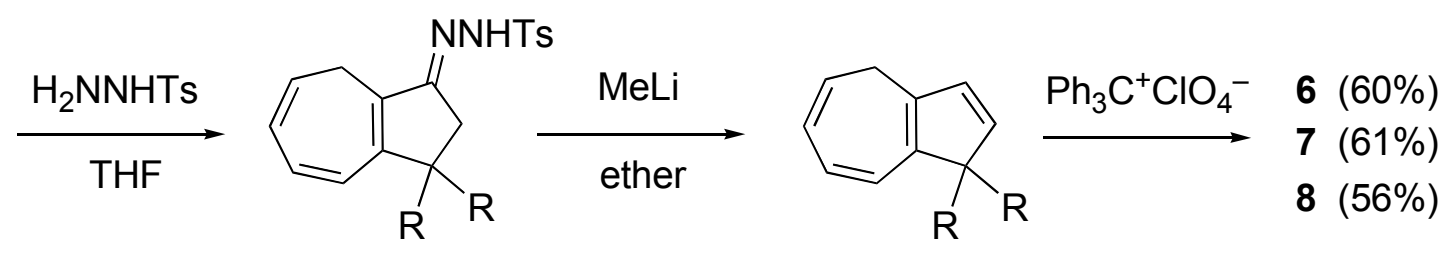

$16(\mathrm{R}=\mathrm{Me})$

17 ( $\mathrm{R}=\mathrm{Et}$ )

$18(\mathrm{R}=\mathrm{Pr})$
$19(\mathrm{R}=\mathrm{Me}, 33 \%$ based on 13$)$

20 ( $R=$ Et, 39\% based on 14)

$21(\mathrm{R}=\mathrm{Pr}, 20 \%$ based on 15) 
Scheme 2

$9 \underset{\mathrm{TMSOTf}}{\stackrel{\mathrm{Pr}}{\mathrm{MeO}}}$ 
Figure 1. UV spectra of the cations 1-3 in acetonitrile. 


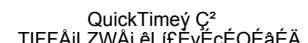

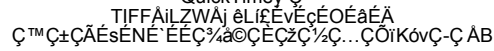

Figure 2. UV spectra of the cations 6-8 in acetonitrile. 
V vs SCE

Figure 3. Cyclic voltammograms of the cations 6-8 in an acetonitrile solution containing $0.1 \mathrm{M}$ tetrabutylammonium perchlorate. 